\title{
环型谐振微环光波导链中光群速度的控制
}

\author{
田 赫* 掌蕴东 王 楠 袁 萍 \\ (哈尔滨工业大学光电子技术研究所, 哈尔滨 $150080 . *$ 联系人, E-mali: tianhe176176@163.com)
}

\begin{abstract}
摘要 利用传输矩阵法对环形谐振微环光波导链进行了理论分析, 得到其透射谱, 其谐振频率与微环 链的曲率无关; 并证明改变微环链的曲率可使色散曲线产生平移. 这样在微环链的谐振频率处可以通 过改变微环链的曲率来调节其群速度. 这种结构在光通信领域有潜在的应用, 包括光缓存器、光信号延 迟器等.
\end{abstract}

关键词 群速度 环型链 微环光波导 传输矩阵法

近年来微波导技术发展迅速，微波导技术中的 调制、解调、光信号传输等已日益成熟，人们更多关 注微波导的一些特殊的结构. 微环结构具有结构简 单、成本低、集成度高等优点, 制作材料甚至可以是 聚合物 ${ }^{[1]}$, 可与直波导耦合, 也容易实现环间耦合形 成微环链. 目前对于这种微环结构在光通信领域的 研究包括滤波 ${ }^{[2 \sim 4]}$ 、波分复用 ${ }^{[5,6]}$ 、波长变换 ${ }^{[7]}$ 、调制 ${ }^{[8]}$ 和开关 ${ }^{[9]}$ 等, 也有应用于激光方面的相关报道 ${ }^{[10]}$. 由于微环的谐振不需要腔面或光棚来提供光反馈, 因此十分有利于与其他光电子元器件的单片集成, 易于制成光学芯片, 是当前光波导技术发展的重要 方向之一, 其在光通信、光学传感及量子光学领域有 重要的应用前景.

微环链式结构在光通信及光信息处理等方面具 有重要意义, 对其研究已经有多篇报道 ${ }^{[11,12]}$, 处理方 法也不尽相同. 利用紧束缚理论 [13]可得到其中传输 电场的本征值, 通过求布洛赫形式的独立谐振模, 得 到微环链色散关系, 但是这种方法比较复杂, 处理问 题比较困难. 而传输矩阵法则较为简便、直观, 只需 求解传输矩阵同样可求得微环链式结构的色散关系, 可证明这两种方法是等价的 ${ }^{[14]}$. 本文利用传输矩阵 法研究环型微环链结构中光群速度的控制.

\section{1 模型及其色散关系}

以 5 个微环为例, 模型结构如图 1 , 微环均匀的 分布在中心 $O$ 的周围, 相邻微环的圆心与 $O$ 的连线 长为 $R_{0}$ 且夹角为 $\theta$, 微环半径均为 $R, R$ 远小于 $R_{0}$. 微 环围绕中心 $O$ 形成了一个理想的圆形, 这一环型特 性可以用夹角 $\theta$ 来表征, 如果令 $\theta \rightarrow 0$, 则变为一般 的直微环链. 微环链的末端与输入输出波导相耦合,
光传播和耦合方向如箭头所示.

一般说来, 我们需要较多的微环来增加微环链的 色散特性, 如图 2. 求解微环链的群速度实际上归结为 求微环链的色散关系. 下面我们利用传输矩阵法求

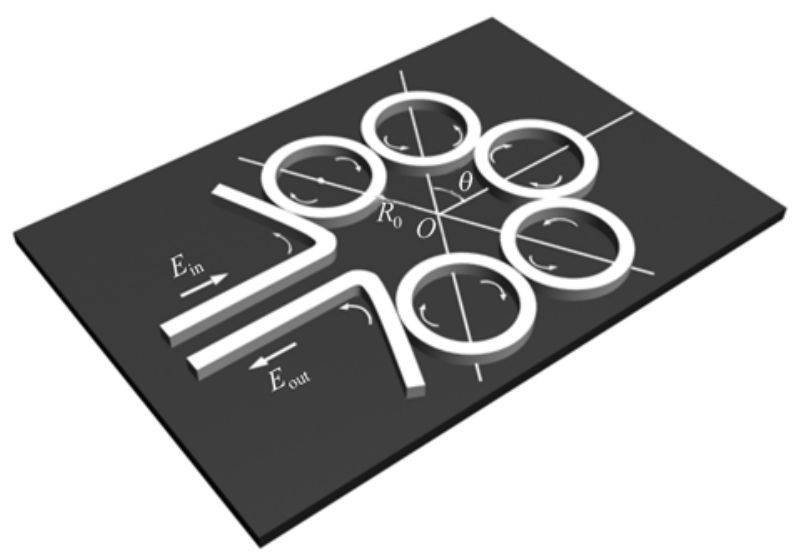

图 15 个微环构成的环型微环链

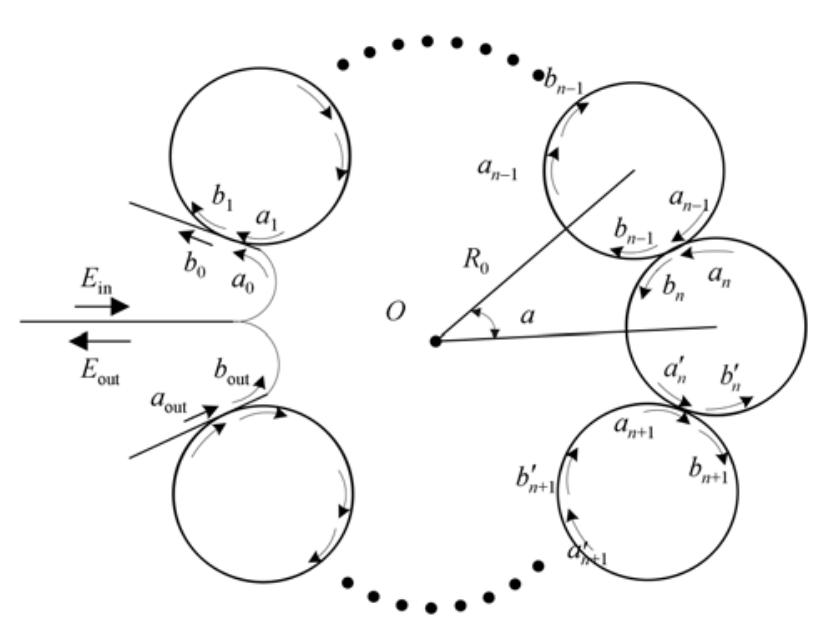

图 2 环型微环链及其振幅矢量 
其色散关系, 仅需对光场振幅进行处理, 求解传输矩 阵. 图 2 中 $\left[\begin{array}{l}a_{n} \\ b_{n}\end{array}\right]$ 和 $\left[\begin{array}{l}a_{n}^{\prime} \\ b_{n}^{\prime}\end{array}\right](n=1,2,3 \cdots)$ 为振幅矢量.

考虑环间耦合损耗为零的理想情况, 这样相邻 微环的耦合可描述为 ${ }^{[15]}$ :

$$
\begin{gathered}
{\left[\begin{array}{l}
a_{n} \\
b_{n}
\end{array}\right]=C\left[\begin{array}{l}
a_{n-1}^{\prime} \\
b_{n-1}^{\prime}
\end{array}\right],} \\
C=i / \varepsilon\left[\begin{array}{cc}
\sqrt{1-\varepsilon^{2}} & -1 \\
1 & -\sqrt{1-\varepsilon^{2}}
\end{array}\right],
\end{gathered}
$$

上式中 $C$ 为归一化的耦合矩阵, $\varepsilon$ 为振幅耦合系数, 在耦合损耗为零的条件下 $\varepsilon$ 为实数且 $0 \leqslant \varepsilon \leqslant 1$.

光波在微环内的传播可用矩阵描述, 在实际中 微环内光损耗主要为传输损耗和弯曲损耗 ${ }^{[16]}$, 这两 者的损耗系数典型值为 $10^{-5} \mathrm{~cm}^{-1}$ 的量级 ${ }^{[17]}$, 在微环 总长度很小时(微环半径为几十 $\mu \mathrm{m}$ ) 光损耗也是很小 的, 为简化计算我们假设光损耗为零, 这样则有:

$$
\begin{gathered}
{\left[\begin{array}{l}
a_{n-1}^{\prime} \\
b_{n-1}^{\prime}
\end{array}\right]=T\left[\begin{array}{l}
a_{n-1} \\
b_{n-1}
\end{array}\right], \quad\left[\begin{array}{l}
a_{n}^{\prime} \\
b_{n}^{\prime}
\end{array}\right]=T^{\dagger}\left[\begin{array}{l}
a_{n} \\
b_{n}
\end{array}\right] ;} \\
T=\left[\begin{array}{cc}
0 & \mathrm{e}^{-\alpha(\pi+\theta)} \\
\mathrm{e}^{\alpha(\pi+\theta)} & 0
\end{array}\right] .
\end{gathered}
$$

上式中 $\alpha=i \frac{n \omega R}{c}, n$ 为光波的有效折射率系数, $\omega$ 为光波角频率, $c$ 为真空中光速, $T^{\dagger}$ 是 $T$ 的复共轭矩阵. 这样相邻环间的场振幅矢量就可用矩阵 $C$ 和 $T$ 联系起来,

$$
\chi_{n+1}=C T^{\dagger} \chi_{n-1}, \quad \chi_{n}=\left[\begin{array}{l}
a_{n} \\
b_{n}
\end{array}\right] .
$$

从另一个角度来讲, 在微环是完全相同的情况 下, 任一组场振幅矢量都可经相同的传输、耦合过程 得到下一组振幅矢量, 所以场是周期性的 ${ }^{[14]}$, 应用 布洛赫定理有

$$
\chi_{n+1}=\mathrm{e}^{-i K A} \chi_{n-1},
$$

式中 $A$ 为晶格常数, 对应两组矢量之间的距离, 在 $\theta$ 很小的情况下, $A \approx 4 R, K$ 为光波在波导中的波数, 由 (3)式与(4)式可得

$$
\mathrm{e}^{-2 \alpha \theta}+\frac{2}{\varepsilon} \sin \left(\frac{n \omega R \pi}{c}\right) \mathrm{e}^{-\alpha \theta} \mathrm{e}^{-i K A}+\mathrm{e}^{-i 2 K A}=0 .
$$

利用单环谐振条件 $[18,19] \frac{n R}{c}=\frac{m}{\Omega}$ 求解(5)式, $\Omega$ 为
谐振角频率, $m$ 为整数; 并对 $\sin \left(\frac{\omega}{\Omega} m \pi\right)$ 做一阶近似 得

$$
\begin{aligned}
& -\sin \frac{2 \omega m \theta}{\Omega}+\frac{2}{\varepsilon}\left(\frac{\omega}{\Omega}\right) m \pi\left(\cos K A \cdot \sin \frac{\omega m \theta}{\Omega}\right. \\
& \left.+\sin K A \cdot \cos \frac{\omega m \theta}{\Omega}\right)=\sin 2 K A .
\end{aligned}
$$

上式即为这种环型微环链结构的色散关系方程.

\section{2 数值计算及讨论}

首先我们计算这种结构的透射谱, 我们需要知 道微环链的曲率是否影响其谐振频率, 因为微环链 的群速度一定程度上依赖于谐振频率. 考虑谐振条 件 $\frac{n R}{c}=\frac{m}{\Omega}$, 选取 $R=25 \mu \mathrm{m}, n=15, \Omega=12.16 \times 10^{14}$ $\mathrm{rad} / \mathrm{s}, m=152$. 图 3 为当夹角 $\theta$ 取 $0 \sim 0.05 \mathrm{rad}$ 时环型 微环链的透射谱, 如果光源线宽足够窄, 那么只存在 谐振频率处的一个透射峰, 可以看出透射谱与 $\theta$ 无关, 即改变微环链的曲率不影响其透射谱, 谐振频率依 然在 $\Omega$ 处.

下面我们考察色散曲线随 $\theta$ 的变化, 利用(6)式 进行计算, $\theta$ 取不同值时色散关系曲线如图 4. 可以看 出, 由于微环链的曲率而引入 $\theta$ 并不会改变色散曲线 的形状, 但却导致曲线平移, $\theta$ 值越大即曲率程度越 大, 曲线向右侧平移得越多, $\theta$ 对色散曲线有一定的 调节作用. 在(6)式中很明显 $\theta$ 存在于三角函数中, 说 明这种调节是周期性的, 周期为 $2 \pi \Omega / m \theta$.

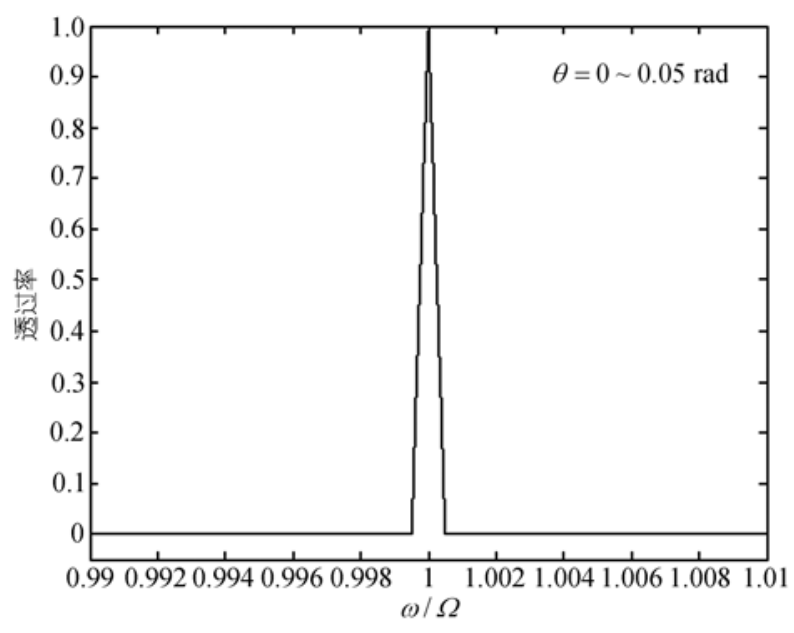

图 3 微环链的透射谱 $(\varepsilon=0.8)$ 


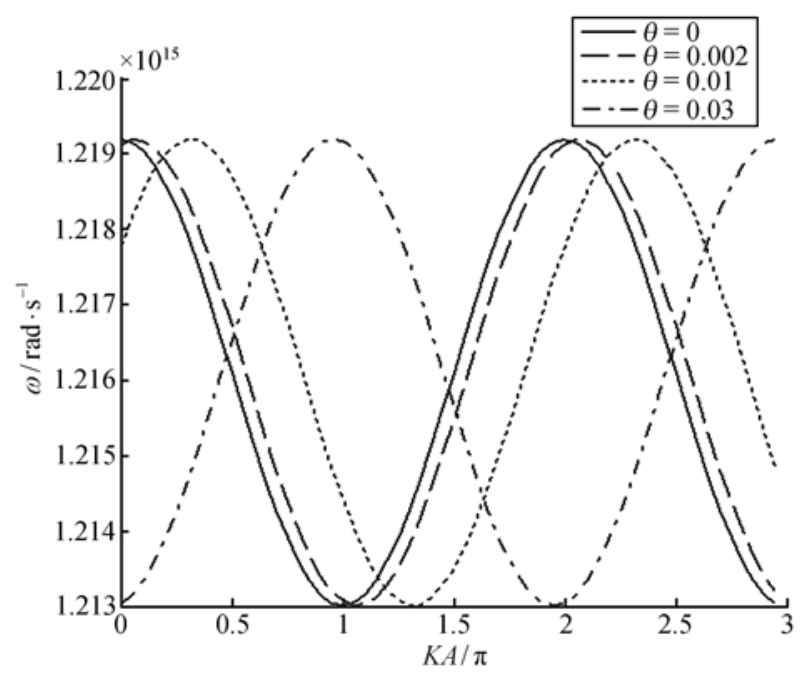

图 $4 \theta$ 取不同值时的色散曲线 $(\varepsilon=0.8, N=15)$

实际上色散曲线的平移可用求传输矩阵 $C T$ 本 征值的方法来更好得说明, 设其本征值为 $\lambda$, 由(3)式 可求得其本征方程为

$$
\mathrm{e}^{-2 \alpha \theta}+\frac{2}{\varepsilon} \sin \left(\frac{n \omega R \pi}{c}\right) \mathrm{e}^{-\alpha \theta} \lambda+\lambda^{2}=0 .
$$

可求得本征值和相应得本征向量分别为

$$
\lambda=\mathrm{e}^{-i \frac{n \omega R}{c} \theta} \mathrm{e}^{ \pm i K A}, \quad \chi=\left[\begin{array}{l}
1 \\
\gamma
\end{array}\right],
$$

其中 $\gamma=\frac{1}{\sqrt{1-\varepsilon^{2}}}\left(\mathrm{e}^{2 \alpha \pi}-i \varepsilon \mathrm{e}^{\alpha \pi} \lambda\right)$.

由(8)式可以看出, 本征向量与 $\theta$ 无关, 在图 4中 表现为色散曲线的形状不随 $\theta$ 的变化而变化; 但同时 本征值却发生了漂移, 漂移量为 $-\frac{n \omega R \theta}{c}$, 在图 4中表 现为色散曲线右向的平移. 由此可以看出在环型微 环链中, 谐振频率是不变的, 适当调节 $\theta$ 的值就可获 得不同的光群速度.

在(8)式中对 $K$ 求导, 可得到群速度 (即 $\left.\frac{\mathrm{d} \omega}{\mathrm{d} K}\right)$ 对 $K$ 的隐函数, 可求其数值解. 图 5(a) 是 $\theta=0.03$ 和 0.04 时 的色散曲线, 对应谐振频率 $\Omega$ 处的 $K A / \pi=1.2$, 如图 5(a)虚线所示. 图 5(b)中 $\theta=0.03$ 时, 对应 $\Omega$ 处群速度 为 $-0.68 \times 10^{7} \mathrm{~m} / \mathrm{s} ; \theta=0.04$ 时, 对应 $\Omega$ 处群速度变为 $1.73 \times 10^{7} \mathrm{~m} / \mathrm{s} . \theta$ 只改变了 0.01 , 而群速度变化了 2.41 $\times 10^{7} \mathrm{~m} / \mathrm{s}$. 这样我们就可以通过改变 $\theta$ 值来选择输出 光波的群速度, 在很大的范围约 $5 \times 10 \mathrm{~m} / \mathrm{s}$ (群速度
最大为 $2.52 \times 10^{7} \mathrm{~m} / \mathrm{s}$, 最小为 $-2.52 \times 10^{7} \mathrm{~m} / \mathrm{s}$ ) 达到控 制群速度的目的.
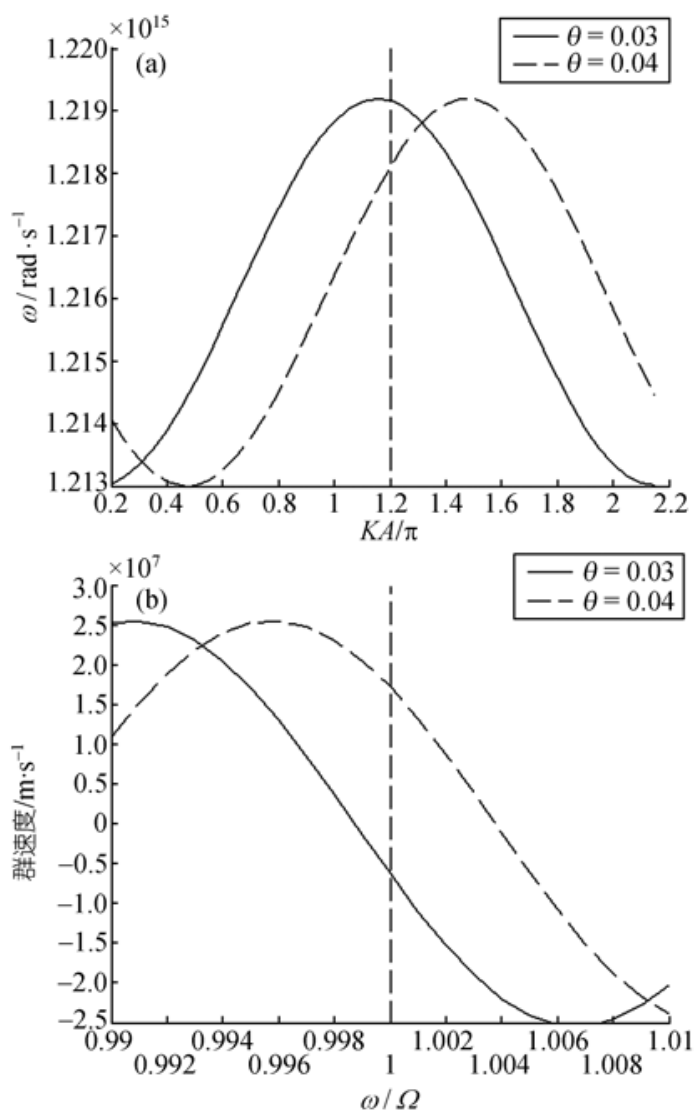

图 5 色散曲线(a)和群速度(b) $(\varepsilon=0.8, N=15)$

\section{3 结论}

针对谐振微环光波导链的色散特性, 采用环型 微环链结构, 利用传输矩阵法建立了其理论模型, 并 进行了数值计算. 环形微环链的曲率可使色散曲线 产生平移, 但不改变色散曲线的形状; 同时微环链的 谐振频率是固定的, 与微环链的曲率无关, 这样就可 通过改变微环链的曲率控制光的群速度. 这种结构 在光通信中有潜在的应用, 包括光缓存器、光信号延 迟、光寄存器等; 也可将这种结构应用到传感方面, 将其与物体结合, 可通过测量群速度的大小, 探测物 体的微小形变.

\section{参考文献}

1 Poon J K S, Zhu L, Derose G A, et al. Polymer microring coupled-resonator optical waveguides. J Lightwave Technol, 2006, 24(4): $1843-1849$ [DOI]

2 Barwicz T, Watts M R, Smith H I. Fabrication of add-drop filters 
based on frequency-matched microring resonators. J Lightwave Technol, 2006, 24(4): 2207-2218[DOI]

3 Okamoto H, Haraguchi M, Okamoto T, et al. Filtering characteristic of a microring resonator with a gap. Electron Commun Jpn, 2006, 89(5): 25-32

4 Djordjev K D, Lin C K, Zhu J T, et al. Folded-cavity resonators as key elements for optical filtering and low-voltage electroabsorption modulation. J Lightwave Technol, 2006, 24(9): $3464-$ $3470[\mathrm{DOI}]$

5 Suzuki S, Hatakeyama Y, Kokubun Y, et al. Precise control of wavelength channel spacing of microring resonator add-drop filter array. J Lightwave Technol, 2002, 20(4): 745-749[DOI]

6 Vorckel A, Monster M, Henschel W, et al. Asymmetrically coupled silicon-on-insulator microring resonators for compact add-drop multiplexers. IEEE Photon Technol Lett, 2003, 15(7): 921923[DOI]

7 Absil P P, Hryniewicz J V, Little B E, et al. Wavelength conversion in GaAs micro-ring resonators. Opt Lett, 2000, 25(8): 554$556[\mathrm{DOI}]$

8 Rabiei P, Steier W H, Zhang C, et al. Polymer micro-ring filters and modulators. J Lightwave Technol, 2002, 20: 1968-1971[DOI]

9 Van V, Ibrahim T A, Ritter K, et al. All-optical nonlinear switching in GaAs-AlGaAs microring resonators. IEEE Photon Technol Lett, 2002, 14: 74-76[DOI]

10 Baktur R, Pearson L W, Ballato J. Theoretical determination of lasing resonances in a microring. J Appl Phys, 2007, 101(4):

\section{2[DOI]}

$11 \mathrm{Xu}$ Y, Lee R K, Yariv A. Propagation and second-harmonic generation of electromagnetic waves in a coupled-resonator optical waveguide. J Opt Soc Am B, 2000, 77: 387-400[DOI]

12 Stefanou N, Modinos A. Impurity bands in photonic insulators. Phys Rev B, 1998, 57: 12127-12133[DOI]

13 Yariv A, Xu Y, Lee R K, et al. Coupled-resonator optical waveguide: a proprosal and analysis. Opt Lett, 1999, 24: 711-713

14 Joyce K S, Scheuer P J, Mookherjea S, et al. Matrix analysis of microring coupled-resonator optical waveguides. Optics Express, 2004, 12: 90-101[DOI]

15 Lee H S, Choi C H, Park D G, et al. A nonunitary transfer matrix method for practical analysis of racetrack microresonator waveguide. IEEE Photon Technol Lett, 2004, 16: $1086-$ 1090[DOI]

16 Choi J M, Lee R K, Yariv A. Control of critical coupling in a ring resonator-fiber configuration: Application to wavelength-selective swithing, modulation, amplification and oscillation. Opt Lett, 2001, 26: $1236-1245$ [DOI]

17 Marcatili E A J. Bends in optical dielectric guides. Bell Syst Tech J, 1996, 48: 2103-2108

18 Heebner J E, Boyd R W. Enhanced all-optical switching by use of a nonlinear fiber ring resonator. Opt Lett, 1999, 24: 847-852

19 Vorckel A, Monster M, Henschel W, et al. Asymmetrically coupled silicon-on-insulator microring resonators for compact add-drop multiplexers. IEEE Photon Technol Lett, 2002, 15: 921—928[DOI] 